TRANSACTIONS OF THE

AMERICAN MATHEMATICAL SOCIETY

Volume 356, Number 11, Pages 4465-4474

S 0002-9947(04)03443-9

Article electronically published on January 13, 2004

\title{
SPÉCIALISATION DE LA $R$-ÉQUIVALENCE POUR LES GROUPES RÉDUCTIFS
}

\author{
PHILIPPE GILLE
}

\begin{abstract}
RÉSUMÉ. Soit $G / k$ un groupe réductif défini sur un corps $k$ de caractéristique distincte de 2 . On montre que le groupes des classes de $R$-équivalence de $G(k)$ ne change pas lorsque l'on passe de $k$ au corps des séries de Laurent $k((t))$, c'est-à-dire que l'on a un isomorphisme naturel $G(k) / R \stackrel{\sim}{\longrightarrow} G(k((t))) / R$.

ABSTRACT. Let $G / k$ be a reductive group defined over a field of characteristic $\neq 2$. We show that the group of $R$-equivalence for $G(k)$ is invariant by the change of fields $k((t)) / k$ given by the Laurent series. In other words, there is a natural isomorphism $G(k) / R \stackrel{\sim}{\longrightarrow} G(k((t))) / R$.
\end{abstract}

La $R$-équivalence est une relation d'équivalence sur les points rationnels d'une variété algébrique introduite par Manin [M] et étudiée par Colliot-Thélène et Sansuc pour les tores algébriques et leurs compactifications [CTS1, par l'auteur pour les variétés de groupes G] et par Kollár pour les variétés géométriquement rationnellement connexes [K1, [K2]. Soit $X / k$ une variété algébrique définie sur un corps $k$. La $R$-équivalence est la relation d'équivalence sur l'ensemble des points rationnels $X(k)$ de $X$ engendrée par la relation élémentaire suivante: deux points $x$ et $y$ de $X(k)$ sont dits directement $R$-équivalents s'il existe une $k$-application rationnelle $\phi$ de la droite projective $\mathbb{P}_{k}^{1}$ dans $X$, définie en 0 et 1 , telle que $\phi(0)=x$ et $\phi(1)=y$.

Soient maintenant $A$ un anneau de valuation discrète, de corps résiduel $k$ et de corps des fractions $F$. On a le résultat suivant de spécialisation pour la $R$ équivalence.

Proposition 0.1 (Madore, non publié). Soit $\mathfrak{X} / \operatorname{Spec}(A)$ un schéma projectif de fibre spéciale $X / k$. Alors la spécialisation $\mathfrak{X}(A)=\mathfrak{X}(F) \rightarrow X(k)$ induit une application

$$
s p: \mathfrak{X}(F) / R \longrightarrow X(k) / R \text {. }
$$

Le but de cet article est d'utiliser cette proposition pour définir une application analogue pour les schémas en groupes réductifs.

Théorème 0.2. On suppose $\operatorname{car}(k) \neq 2$. Soit $\mathfrak{G} / A$ un $A$-groupe réductif de fibre spéciale $G / k$ (connexe). On note $g \mapsto \bar{g}$ l'application de spécialisation $\mathfrak{G}(A) \rightarrow$ $G(k)$.

a) Il existe un unique morphisme de spécialisation fonctoriel en $A$ et $\mathfrak{G}$

$$
s p: \mathfrak{G}(F) / R \longrightarrow G(k) / R,
$$

tel que $[s p(g)]=[\bar{g}]$ pour tout $g \in \mathfrak{G}(A)$.

Received by the editors April 9, 2003 and, in revised form, May 9, 2003.

2000 Mathematics Subject Classification. Primary 20G15, 14L40.

(C)2004 American Mathematical Society 
b) Si A est hensélien (par exemple complet), il existe un morphisme $\varphi: G(k) \rightarrow$ $\mathfrak{G}(F) / R$ tel que sp $\circ \varphi$ est la surjection canonique $G(k) \rightarrow G(k) / R$.

c) Si A est hensélien et a un corps de coefficients (e.g. si $\operatorname{car}(k)=0)$, alors le morphisme sp est un isomorphisme.

On obtient ainsi le résultat suivant, conjecturé par J.-L. Colliot-Thélène, qui généralise le cas connu des tores (§3.2).

Corollaire 0.3. Soit $G / k$ un groupe réductif (connexe) défini sur un corps de caractéristique distincte de 2. Alors on a une isomorphisme naturel

$$
G(k) / R \stackrel{\sim}{\longrightarrow} G(k((t))) / R .
$$

Cet article est motivé par l'article de Chernousov et Merkurjev où l'application de spécialisation est définie dans le cas particulier du groupe de Clifford d'une forme quadratique ([CM], $\S 4$, p. 521). Ainsi, ce résultat général permet de simplifier, dans une certaine mesure, des points techniques de cet article.

Il s'applique aussi au cas du groupe $S L_{1}(D)$ d'une algèbre simple centrale $D / k$ pour lequel on retrouve la flèche de spécialisation habituelle pour le groupe $S L_{1}(D)(k) / R$ définie avec la $K$-théorie algébrique via la théorème de Voskresenkiǐ $S K_{1}(D) \stackrel{\sim}{\longrightarrow} S L_{1}(D)(k) / R[\nabla]$ et on retrouve également la propriété $S K_{1}(D)=$ $S K_{1}\left(D_{k((t))}\right)$.

Le passage de la proposition 0.1 au théorème 0.2 se fait en deux étapes. La première consiste en une réduction au cas anisotrope et la seconde en la construction de compactifications explicites de $\mathfrak{G}$, notamment la compactification magnifique de De Concini et Procesi, expliquant l'hypothèse faite sur la caractéristique de $k$ dans le théorème.

\section{Compactifications et $R$-ÉQuivalence}

1.1. Compactification des groupes algébriques linéaires anisotropes. Le but de cette section est de caractériser l'anisotropie des groupes algébriques linéaires avec les compactifications. On rappelle que le $k$-groupe réductif $G$ (connexe) est isotrope s'il contient un $k$-tore déployé non trivial.

Proposition 1.1. On suppose que $G / k$ admet une compactification lisse $X / k$. Alors les assertions suivantes sont équivalentes:

i) $G$ est anisotrope,

ii) $G(k)=X(k)$.

L'ingrédient principal utilisé dans la proposition est le théorème suivant de Bruhat-Tits-Rousseau.

Théorème $1.2(\mathrm{BrT}$, voir aussi $[\mathrm{P}]$ et $[\mathrm{T}$, fin de la section 2, page 663). On suppose $A$ hensélien. Les assertions suivantes sont équivalentes:

i) $G / k$ est anisotrope,

ii) $\mathfrak{G}_{F}$ est anisotrope,

iii) $\mathfrak{G}(A)=\mathfrak{G}(F)$.

Démonstration de la proposition [1.1]. Suivant le théorème précédent, il est loisible de remplacer le corps $k$ par le corps $k(t)$, et ainsi supposer le corps $k$ infini.

i) $\Longrightarrow$ ii): On suppose que $G$ contient un sous-groupe $\mathbb{G}_{m, k}$. L'inclusion $\mathbb{G}_{m, k}$ se prolonge en un morphisme $f: \mathbb{P}_{k}^{1} \rightarrow X$. Si $f(0), f(\infty) \in G(k)$, alors $f$ est à valeurs 
dans la variété affine $G$, donc est constant, ce qui est une contradiction. On conclut que $X(k) \backslash G(k) \neq \emptyset$.

ii) $\Longrightarrow$ i): On note $\partial X=X \backslash G$ le bord de $X$. Soit $x \in X(k) \backslash G(k)$; on note $O_{X, x}$ l'anneau local et $\widehat{O_{X, x}}$ son complété. Il existe $T_{1}, \ldots, T_{d} \in \widehat{O_{X, x}}$ tels que $\widehat{O_{X, x}}$ soit $k$-isomorphe à $k\left[\left[T_{1}, \ldots, T_{d}\right]\right]$. Quitte à effectuer un changement linéaire de coordonnées, on peut supposer que le sous-schéma $Y \operatorname{de} \operatorname{Spec}\left(\widehat{O_{X, x}}\right)$ défini par les équations

$$
T_{2}=T_{3}=\cdots=T_{d}=0
$$

satisfait

$$
Y \times_{X} \partial X=\{x\}
$$

avec la convention $Y=X$ si $d=1$. On considère le morphisme

$$
\eta: \operatorname{Spec}\left(k\left[\left[T_{1}\right]\right]\right) \rightarrow X
$$

donné par le quotient $\widehat{O_{X, x}} \rightarrow k\left[\left[T_{1}, \ldots, T_{d}\right]\right] /\left(T_{2}, T_{3}, \ldots, T_{d}\right) \stackrel{\sim}{\longrightarrow} k\left[\left[T_{1}\right]\right]$, ou en termes géométriques la droite formelle d'équations $T_{2}=T_{3}=\cdots=T_{d}$ (appartenant à $Y$ ). On a $\eta(0)=x$ donc $\eta \notin G\left(k\left[\left[T_{1}\right]\right]\right)$. On note

$$
\eta_{\text {gen }}: \operatorname{Spec}\left(k\left(\left(T_{1}\right)\right)\right) \stackrel{\eta}{\rightarrow} \operatorname{Spec}\left(k\left[\left[T_{1}\right]\right]\right) \rightarrow X
$$

le composé. Par construction, $\eta_{\text {gen }} \in G\left(k\left(\left(T_{1}\right)\right)\right)$. Par suite,

$$
G\left(k\left[\left[T_{1}\right]\right]\right) \neq G\left(k\left(\left(T_{1}\right)\right)\right),
$$

donc le théorème 1.2 montre que le groupe $G / k$ est isotrope.

Ce résultat concerne le cas de caractéristique nulle où il existe une compactification lisse de $G$ d'après le théorème d'Hironaka, et aussi le cas des groupes adjoints en caractéristique distincte de 2 car on dispose alors des compactifications magnifiques de De Concini et Procesi ([CS], §3).

Lemme 1.3. On suppose $\operatorname{car}(k) \neq 2$ et $A$ hensélien. On suppose que $G / k$ est anisotrope. Alors il existe une compactification $X / k$, non nécessairement lisse, satisfaisant

$$
G(k)=X(k) .
$$

Si de plus $\mathfrak{G} / A$ est un $A$-schéma en groupes réductif de fibre spéciale $G$, on peut choisir la compactification $G \subset X$ se relevant en $\mathfrak{G} \subset \mathfrak{X}$ avec $\mathfrak{X}$ propre sur $\operatorname{Spec}(A)$.

Démonstration. On note $\mathfrak{G}_{a d}$ le groupe adjoint de $\mathfrak{G}$ ([SGA3], exp. XXII, §4.3.6) et $\mathfrak{T}$ le tore coradical de $\mathfrak{G}$ ( $i b i d, \S 6.2 .1)$. La proposition 6.2 .4 de loc cit entraîne que l'on a une isogénie centrale

$$
1 \rightarrow \mu \rightarrow \mathfrak{G} \stackrel{\lambda}{\rightarrow} \mathfrak{T} \times{ }_{A} \mathfrak{G}_{a d} \rightarrow 1
$$

où $\mu$ est un $A$-schéma en groupes fini de type multiplicatif. Suivant la preuve du lemme 12 de [CTS1, il existe une $k$-compactification projective $T^{c}$ de $T$ telle que $T(k)=T^{c}(k)$, rappelons sa construction. On choisit un plongement $T \subset$ $\left(R_{k^{\prime} / k} \mathbb{G}_{m}\right)^{n}$ où $k^{\prime} / k$ est une extension séparable de corps, $R_{k^{\prime} / k}$ désignant le foncteur de restriction des scalaires à la Weil ([BLR], §7.6). La compactification $T^{c}$ est l'adhérence de $T$ dans $\left(R_{k^{\prime} / k}\left(\mathbb{P}_{k^{\prime}}^{1}\right)\right)^{n}$. On relève cette construction en prenant un plongement

$$
\mathfrak{T} \subset\left(R_{A^{\prime} / A} \mathbb{G}_{m}\right)^{n} \subset\left(R_{A^{\prime} / A} \mathbb{P}_{A}^{1}\right)^{n},
$$


où $A^{\prime} / A$ est l'extension non ramifiée d'anneaux henséliens relevant $k^{\prime} / k$. Vu que le tore $\mathfrak{T} / F$ est anisotrope, on a $\mathfrak{T} \subset\left(R_{A^{\prime} / A}^{1} \mathbb{G}_{m}\right)^{n}$. On note $\mathfrak{Y}$ l'adhérence de $\mathfrak{T}$ dans $\left(R_{A^{\prime} / A}\left(\mathbb{P}_{A}^{1}\right)\right)^{n}$ et on pose $Y=\mathfrak{Y} \times{ }_{A} k$. Suivant loc cit, on a

Lemme 1.4. $T(k)=Y(k)$.

Passons à la partie adjointe. Suivant le théorème 3.13 de [CS], il existe une $A$ compactification projective $\mathfrak{Z}$ de $\mathfrak{G}_{a d}$ telle que $Z:=\mathfrak{Z} \times{ }_{A} k$ est une $k$-compactification de De Concini-Procesi de $G_{a d}$. Suivant la proposition 1.1, on a $G_{a d}(k)=Z(k)$. On définit le schéma $\mathfrak{X}$ comme le normalisé par l'extension $F(\mathfrak{G}) / F\left(\mathfrak{T} \times_{A} \mathfrak{G}_{a d}\right)$ de $\mathfrak{Y} \times \mathfrak{Z}$. On a alors un morphisme fini $\mathfrak{X} \rightarrow \mathfrak{Y} \times_{A} \mathfrak{Z}$ prolongeant $\lambda$. Par suite, $\mathfrak{X}$ est une $A$-compactification de $\mathfrak{G}$. Comme $\mathfrak{G}$ est un schéma normal, on a

$$
\mathfrak{G}=\left(\mathfrak{T} \times_{A} \mathfrak{G}_{a d}\right) \times_{\left(\mathfrak{Y} \times{ }_{A} \mathfrak{Z}\right)} \mathfrak{X} .
$$

Par suite, on a $G=\left(T \times G_{a d}\right) \times_{(Y \times Z)} X$ où $X=\mathfrak{X} \times_{A} k$, et on conclut que $G(k)=X(k)$.

1.2. Réduction de la $R$-équivalence au cas anisotrope. On rappelle que la $R$-équivalence sur le groupe $G / k$ est compatible à la structure de groupe de $G(k)$, ainsi l'ensemble $R G(k)$ des éléments $R$-équivalents à $e \in G(k)$ est un sous-groupe normal de $G(k)$ et $G(k) / R=G(k) / R G(k)$ ([G], lemme II.1.1). De plus, deux éléments $R$-équivalents de $G(k)$ le sont élémentairement (ibid).

Proposition 1.5 (proposition 11 de [CTS1]). On suppose $k$ infini. Soit $H / k$ un groupe algébrique linéaire connexe supposé réductif si $k$ n'est pas parfait et soit $U \subset H$ un ouvert non vide. Alors on a une bijection $U(k) / R \stackrel{\sim}{\longrightarrow} H(k) / R$.

Corollaire 1.6. On suppose $k$ infini. Soit $M / k \subset H / k$ un sous-groupe algébrique linéaire d'un $k$-groupe algébrique linéaire $H / k$. On suppose qu'il existe une variété $V / k$, ouvert non vide d'un espace affine, et un $k-$ morphisme $M \times V \hookrightarrow H$ induisant une $k$-immersion ouverte. Alors on a un isomorphisme

$$
M(k) / R \stackrel{\sim}{\longrightarrow} H(k) / R .
$$

Le corollaire résulte immédiatement de la proposition et de l'identité

$$
(M \times V)(k) / R=M(k) / R \times V(k) / R=M(k) / R .
$$

Lemme 1.7. On suppose $k$ infini. Soit $P$ un sous-groupe parabolique de $G, L$ un sous-groupe de Levi de $P$ et $S / k$ le sous-tore maximal déployé de $Z(L)$ (en particulier, si $P$ est minimal, le groupe $L / S$ est anisotrope). Alors pour tout corps $F / k$, on a des isomorphismes

$$
(L / S)(F) / R \stackrel{\sim}{\longleftarrow} L(F) / R \stackrel{\sim}{\longrightarrow} G(F) / R .
$$

Démonstration du lemme. Comme $S$ est déployé, le morphisme $f: L \rightarrow L / S$ admet une section rationnelle. On choisit un ouvert non vide $U$ de $L / S$ tel que $f^{-1}(U)=$ $U \times S$. Suivant la proposition, on a des bijections $L(k) / R=(U \times S)(k) / R=$ $U(k) / R=(L / S)(k) / R$ et le morphisme $L(k) \rightarrow(L / S)(k)$ est un isomorphisme. Le morphisme $G \rightarrow G / P$ admet une section rationnelle, on choisit un ouvert $V$ de $G / P$ et une section $s: V \rightarrow G$ de $G \rightarrow G / P$. Le $k$-morphisme $P \times V \rightarrow G$ donné par $(p, v) \mapsto p . s(v)$ est une immersion ouverte. Comme $G / P$ est une variété rationnelle ([Bo], th. 21.20, p. 240), le corollaire montre que $P(k) / R \rightarrow G(k) / R$ est un isomorphisme. Enfin, le morphisme $L \rightarrow P$ s'étend en un isomorphisme de variétés 
$L \times R_{u}(P) \stackrel{\sim}{\longrightarrow} P$. Le radical unipotent $R_{u}(P)$ est un $k$-groupe unipotent déployé, donc est isomorphe à un espace affine. On conclut que l'on a des isomorphismes $L(k) / R \stackrel{\sim}{\longrightarrow} P(k) / R \stackrel{\sim}{\longrightarrow} G(k) / R$.

\subsection{Cas particulier de la caractéristique nulle.}

Proposition 1.8. On suppose $\operatorname{car}(\mathrm{k})=0$. Soit $X / k$ une compactification lisse de $G / k$. Alors l'application $G(k) / R \rightarrow X(k) / R$ est bijective.

L'hypothèse de caratéristique nulle intervient dans la résolution forte canonique des singularités de Bierstone-Milman [BM] (voir aussi Encinas-Hauser [EH]) précisant le théorème d'Hironaka $[\mathrm{H}$; la toute puissance de ce théorème coupe court à une étude plus fine des compactifications de groupes algébriques par des méthodes spécifiques au contexte, notamment la théorie des éventails et des singularités toriques.

Lemme 1.9. On suppose $\operatorname{car}(\mathrm{k})=0$. Soit $H / k$ un groupe algébrique linéaire. Alors il existe une $k$-compactification projective lisse $Y$ de $H$ munie d'une action du groupe $H \times H$ prolongeant l'action de $H \times H$ sur $H$ définie par $\left(h_{1}, h_{2}\right) . h=h_{1} h h_{2}^{-1}$.

Démonstration. Soit $H \subset G L_{n}$ une représentation linéaire de $H$. Le morphisme évident $G L_{n} \rightarrow G L_{n+1}$ fait de $H$ un sous-groupe de $P G L_{n+1}$. Le groupe $P G L_{n+1}$ est un ouvert de l'espace projectif $\mathbb{P}\left(M_{n+1}\right)$ qui est muni d'une action de $P G L_{n+1} \times$ $P G L_{n+1}$ prolongeant l'action sur $P G L_{n+1}$. On note $Z / k$ l'adhérence de Zariski de $H$ dans $\mathbb{P}\left(M_{n+1}\right)$; par construction, la variété projective $Z / k$ est munie d'une action de $H \times H$ prolongeant l'action sur $H$. Le théorème de résolution forte des singularités produit une désingularisation $f: Y \rightarrow Z$ satisfaisant les propriétés suivantes $[\mathrm{BM}$ :

i) $Y$ est projective lisse,

ii) $f$ induit un isomorphisme $f^{-1}(H) \stackrel{\sim}{\longrightarrow} H$,

iii) $Y$ est muni d'une action de $H \times H$ et $f$ est $H \times H$-équivariant pour cette action.

Démonstration de la proposition 1.8. Selon le corollaire ii) de la proposition 10 de [CTS1], cette assertion ne dépend pas de la compactification lisse choisie, il suffit donc de la montrer pour une compactification particulière. De plus, selon la proposition 13 du même article, la proposition est connue pour les tores. La démonstration comporte plusieurs étapes. Remarquons tout d'abord qu'elle est de façon évidente stable par produit.

Lemme 1.10. a) Soit $U$ un sous-groupe unipotent normal de G. Si la proposition vaut pour $G / U$, alors elle vaut pour $G$.

b) Soit $S$ un k-tore déployé central de $G$. Si la proposition vaut pour $G / S$, alors elle vaut pour $G$.

c) Soit $P / k$ un sous-groupe parabolique de $G / k$. Si la proposition vaut pour $P$, alors elle vaut pour $G$.

Démonstration du lemme. a) On sait que le morphisme $G \rightarrow G / U$ est scindé, ainsi on a un isomorphisme de $k$-variétés $G=G / U \times U$. En prenant une compactification lisse produit, il suffit de voir que la proposition vaut pour $U$. Or $U$ est une variété $k-$ rationnelle, donc toute compactification lisse $Y$ de $U$ satisfait $Y(k) / R=1$ suivant la proposition 10 de CTS1] et par dévissage au cas de $\mathbb{G}_{a}$, on $U(k) / R=Y(k) / R=1$. 
b) Par récurrence, on peut supposer que $S=\mathbb{G}_{m}$. On note $Y$ une compactification lisse de $G / \mathbb{G}_{m}$. Le fibré inversible $G \rightarrow G / \mathbb{G}_{m}$ se prolonge en un fibré inversible $Y^{\prime} \rightarrow Y$, formé des sections non nulles d'un fibré vectoriel $E \rightarrow Y$. Ainsi, $G$ admet la compactification lisse $\mathbb{P}(E) / Y$. Le même argument que celui de la proposition 10 de CTS1 montre que l'application $\mathbb{P}(E)(k) / R \rightarrow Y(k) / R$, à l'évidence surjective, est bijective. Par hypothèse, on a $\left(G / \mathbb{G}_{m}\right)(k) / R=Y(k) / R$ et donc comme $G(k) / R=\left(G / \mathbb{G}_{m}\right)(k) / R$, on conclut que $G(k) / R=\mathbb{P}(E)(k) / R$.

c) Le $P$-torseur $G \rightarrow G / P$ est localement trivial pour la topologie de Zariski ([Bo], §20.5). Suivant le lemme [1.9, il existe une compactification lisse $Y$ de $P$ qui est $P$-équivariante pour l'action de $P \times P$ sur $P$ donnée par $\left(p_{1}, p_{2}\right) \cdot p=p_{1} p p_{2}^{-1}$. Alors le $P$-torseur $G \rightarrow G / P$ s'étend de façon unique en un morphisme projectif lisse $X \rightarrow G / P$ de fibre $Y$, qui est une compactification lisse de $G$, i.e. on a le diagramme commutatif

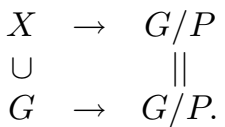

Par hypothèse, on a $P(k) / R=Y(k) / R$, et ainsi vu que les fibres de $X(k) \rightarrow$ $(G / P)(k)$ sont isomorphes à $Y(k)$, l'application $G(k) / R \rightarrow X(k) / R$ est surjective. Etablissons l'injectivité. Soient $h, g$ deux points de $G(k) R$-équivalents dans $X$. Il existe alors des points $x_{0}=h, x_{1}, \ldots, x_{n}=g$ de $X(k)$ tels que $x_{i}$ est directement $R$-équivalent à $x_{i+1}$. Il existe un ouvert $U$ de $G / P$ contenant les images des $x_{i}$ qui trivialise la fibration $G \rightarrow G / P$. Alors $G \times_{G / P} U \stackrel{\sim}{\longrightarrow} P \times U$ et $X \times_{G / P} U \stackrel{\sim}{\longrightarrow}$ $Y \times U$. Par hypothèse, on a $P(k) / R=Y(k) / R$, on en déduit que $h$ et $g$ sont $R$-équivalents.

Ce lemme de dévissage nous ramène donc au cas d'un groupe réductif anisotrope pour lequel on a $G(k)=X(k)$ et $G(k(t))=X(k(t))$ suivant la proposition 1.1. On conclut que $G(k) / R \stackrel{\sim}{\longrightarrow} X(k) / R$.

\section{SpéCiAlisAtion}

On va démontrer dans cette section le théorème principal.

Théorème 2.1. On suppose $\operatorname{car}(k) \neq 2$. Soit $\mathfrak{G} / A$ un $A$-groupe réductif linéaire de fibre spéciale $G / k$ (connexe). On note $g \mapsto \bar{g}$ l'application de spécialisation $\mathfrak{G}(A) \rightarrow G(k)$.

a) Il existe un unique morphisme de spécialisation fonctoriel en $A$ et $\mathfrak{G}$

$$
s p: \mathfrak{G}(F) / R \longrightarrow G(k) / R
$$

tel que $[\operatorname{sp}(g)]=[\bar{g}]$ pour tout $g \in \mathfrak{G}(A)$.

b) Si $A$ est hensélien (par exemple complet), il existe un morphisme $\varphi: G(k) \rightarrow$ $\mathfrak{G}(F) / R$ tel que spo $\varphi$ est la surjection canonique $G(k) \rightarrow G(k) / R$.

c) Si A est hensélien et a un corps de coefficients (e.g. si car $(k)=0)$, alors le morphisme sp est un isomorphisme.

2.1. Définition de l'application de spécialisation. On peut supposer $A$ hensélien. Selon le lemme [1.7 on peut supposer que le groupe $G / k$ est anisotrope. Suivant le théorème de Bruhat-Tits-Rousseau [1.2 on a $\mathfrak{G}(A)=\mathfrak{G}(F)$ et l'unicité de l'application de spécialisation est alors évidente. Le lemme 1.3 montre qu'il existe une $A$-compactification $\mathfrak{X} / A$ de $\mathfrak{G} / A$ telle que $G(k)=X(k)$ où $X=\mathfrak{X} \times{ }_{A} K$. 
Comme $G_{k(t)}$ est anisotrope, on a aussi $G(k(t))=X(k(t))$ d'où un isomorphisme $G(k) / R \stackrel{\sim}{\longrightarrow} X(k) / R$.

Le lemme de Chow (GD], §5.6) montre qu'il existe un ouvert $\mathfrak{U}$ de $\mathfrak{G}$, un $A$ morphisme $\mathfrak{X}^{\prime} \rightarrow \mathfrak{X}$ projectif et surjectif induisant un isomorphisme $f: f^{-1}(\mathfrak{U}) \stackrel{\sim}{\longrightarrow}$ $\mathfrak{U}$ avec $\mathfrak{X}^{\prime}$ est quasi-projectif sur $\operatorname{Spec}(A)$. Ainsi le $A$-schéma $\mathfrak{X}^{\prime}$ est quasi-projectif et propre, donc projectif suivant (ibid, Th. 5.5.3). La proposition 0.1 montre que l'on dispose d'une application de spécialisation

$$
\mathfrak{U}(F) / R \rightarrow \mathfrak{X}^{\prime}(F) / R \stackrel{s p}{\rightarrow} X^{\prime}(k) / R \rightarrow X(k) / R \stackrel{\sim}{\longleftarrow} G(k) / R .
$$

Suivant la proposition 1.5, on a un isomorphisme $\mathfrak{U}(F) / R \stackrel{\sim}{\longrightarrow} \mathfrak{G}(F) / R$, donc le composé ci-dessus définit le morphisme de spécialisation

$$
s p: \mathfrak{G}(F) / R \rightarrow G(k) / R .
$$

2.2. Le cas des tores algébriques. On suppose l'anneau $A$ hensélien jusqu'à la fin de la section 2. Tout d'abord, on va vérifier que le théorème pour les tores algébriques est une conséquence du calcul de la $R$-équivalence pour les tores de Colliot-Thélène et Sansuc [CTS1]. Si $T$ est un $k$-tore, il existe une suite exacte de $k$-tores

$$
1 \rightarrow S \rightarrow E \rightarrow T \rightarrow 1,
$$

où $E$ est un tore quasi-trivial (i.e. un produit de tores $R_{L / k} \mathbb{G}_{m}$ ) et $S$ un $k$-tore flasque, c'est-à-dire satisfaisant $H^{1}\left(L, \operatorname{Hom}\left(\mathbb{G}_{m}, S\right)\right)=0$ pour toute extension $L / k$. Alors le bord $T(k) \rightarrow H^{1}(k, S)$ induit un isomorphisme $T(k) / R \stackrel{\sim}{\longrightarrow} H^{1}(k, S)$.

Proposition 2.2. Soit $\mathfrak{T} / A$ un A-tore algébrique de fibre spéciale T. Alors on a un isomorphisme naturel

$$
\mathfrak{T}(F) / R \stackrel{\sim}{\longrightarrow} T(k) / R
$$

Démonstration. Selon la proposition 1.3.3 de [CTS2], il existe une résolution flasque de $A$-tores

$$
1 \rightarrow \mathfrak{S} \rightarrow \mathfrak{E} \rightarrow \mathfrak{T} \rightarrow 1
$$

bien que nous ne donnions pas ici la définition précise, cela entraîne en particulier suivant (ibid, proposition 1.4) que la fibre spéciale (resp. générique) est une résolution flasque de $T$ (resp. $\left.\mathfrak{T}_{F}\right)$. On a un diagramme commutatif

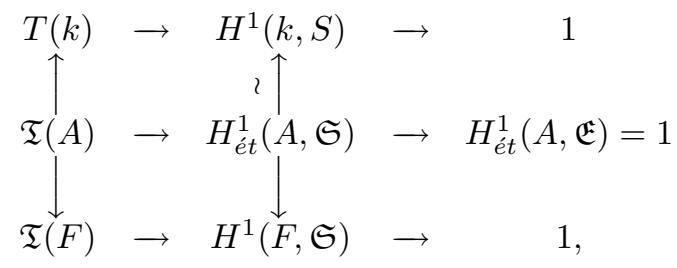

l'isomorphisme $H_{e ́ t}^{1}(A, \mathfrak{S}) \stackrel{\sim}{\longrightarrow} H^{1}(k, S)$ étant le lemme de Hensel ([SGA3], Exp. XXIV, Prop. 8.2.(ii)). Comme $\mathfrak{S} / A$ est flasque, le théorème 2.2.(i) de [CTS2] montre que la restriction $H_{e ̂ t}^{1}(A, \mathfrak{S}) \rightarrow H^{1}(F, \mathfrak{S})$ est surjective. Par ailleurs, cette restriction est injective (ibid, théorème 4.1.(i)), donc on a un isomorphisme

$$
H_{e ̂ t}^{1}(A, \mathfrak{S}) \stackrel{\sim}{\longrightarrow} H^{1}(F, \mathfrak{S}) .
$$

On conclut que la spécialisation $\mathfrak{T}(A) \rightarrow T(k)$ induit un isomorphisme naturel $\mathfrak{T}(F) / R \stackrel{\sim}{\longrightarrow} \mathfrak{T}(k) / R$. 
2.3. Rigidité. On suppose $G$ anisotrope.

Lemme 2.3. Soient $g_{0} \in \mathfrak{G}(A)$ et $g \in \mathfrak{G}(A)$ tel que $\bar{g}=\bar{g}_{0}$ soit un élément semi-simple régulier de $G(k)$. Alors il existe $h \in \operatorname{Ker}(\mathfrak{G}(A) \rightarrow \mathfrak{G}(k))$ tel que $g h g_{0}^{-1} h^{-1} \in R \mathfrak{G}(F)$.

Démonstration. Les centralisateurs $Z_{\mathfrak{G}}\left(g_{0}\right) / A$ et $Z_{\mathfrak{G}}(g) / A$ sont des $A$-tores maximaux de $\mathfrak{G} / A$ satisfaisant $Z_{\mathfrak{G}}\left(g_{0}\right) \times_{A} k=Z_{\mathfrak{G}}(g) \times_{A} k$. Suivant le théorème de rigidité de Grothendieck sur les sous-tores des schémas en groupes linéaires [SGA3. exp. IX, cor. 7.3, p. 72], il existe un élément $h \in \mathfrak{G}(A)$ tel que $\bar{h}=1$ et tel que $Z_{\mathfrak{G}}(g) / A=h Z_{\mathfrak{G}}\left(g_{0}\right) h^{-1} / A$. Suivant le cas des tores appliqué à $Z_{\mathfrak{G}}\left(g_{0}\right) / A, h^{-1} g h$ est $R$-équivalent à $g_{0}$.

Lemme 2.4. $\operatorname{Ker}(\mathfrak{G}(A) \rightarrow G(k)) \subset R \mathfrak{G}(F)$.

Démonstration. Si $k$ est fini, le groupe $G / k$ est un tore et donc la proposition 2.2 établit ce cas. On suppose donc $k$ infini, et vu que $R G(k)$ est Zariski-dense dans $G$, on peut choisir un élément semi-simple régulier $\bar{g}_{0} \in R G(k)$, que l'on relève en un élément $g_{0} \in \mathfrak{G}(A)=\mathfrak{G}(F)$. On va voir que l'on peut construire un tel $g_{0}$ satisfaisant de plus $g_{0} \in R \mathfrak{G}(F)$. Dans ce but, on considère le $A$-tore $Z_{\mathfrak{G}}\left(g_{0}\right)$ et vu que $R Z_{G}\left(\bar{g}_{0}\right)(k)$ est Zariski-dense dans $Z_{G}\left(\bar{g}_{0}\right)$, on peut supposer que $\bar{g}_{0} \in R Z_{G}\left(g_{0}\right)(k)$, d'où $g_{0} \in R Z_{\mathfrak{G}}\left(g_{0}\right)(F) \subset R \mathfrak{G}(F)$ suivant la proposition 2.2 Soit $g \in \mathfrak{G}(A)$ satisfaisant $\bar{g}=1$. Suivant le lemme 2.3 appliqué à $g g_{0}$, il existe $h \in G(A)$ tel que $g g_{0} h g_{0}^{-1} h^{-1} \in R \mathfrak{G}(F)$. Comme $g_{0} \in R \mathfrak{G}(F)$, on a $g \in R \mathfrak{G}(F)$. On en déduit que

$$
\operatorname{Ker}(\mathfrak{G}(A) \rightarrow G(k)) \subset R \mathfrak{G}(F) .
$$

Le passage au quotient par $\operatorname{Ker}(\mathfrak{G}(A) \rightarrow G(k))$ produit un morphisme surjectif

$$
\varphi: G(k) \rightarrow \mathfrak{G}(F) / R
$$

tel que le composé $s p \circ \varphi$ soit le morphisme canonique $G(k) \rightarrow G(k) / R$.

Montrons maintenant l'assertion c) où l'on suppose que $k$ se plonge dans $A$. Le lemme de Hensel montre que l'on peut supposer (à isomorphisme près) que $\mathfrak{G}=G \stackrel{s}{\times}_{k} A$. Le morphisme $\varphi$ est donné par l'extension des scalaires de $k$ à $F$ et induit donc un morphisme surjectif $\bar{\varphi}: G(k) / R \rightarrow \mathfrak{G}(F) / R$. Il résulte que sp $: \mathfrak{G}(F) / R \rightarrow G(k) / R$ est un isomorphisme.

\section{Autres DÉmonstrations}

3.1. Rigidité d'après Raghunathan. Cela concerne le cas de l'anneau complet $O=k[[t]]$ de corps des fractions $K=k((t))$. On note $G_{1}(O)=\operatorname{Ker}(G(O) \rightarrow G(k))$.

Proposition 3.1 ( $[\mathrm{R}]$, proposition 1.3). Il existe une famille finie de $k$-tores $k-$ rationnels $\left(\widetilde{T}_{i}\right)_{i=1, \ldots, n}$, des $k$-morphismes de groupe $f_{i}: \widetilde{T}_{i} \rightarrow G(i=1, \ldots, r)$, et des éléments $\left(g_{i}\right)_{i=1, \ldots, n}$ tels que l'application

$$
\begin{array}{ccc}
\prod_{i=1, \ldots, n} \widetilde{T}_{i, 1}(O) & \rightarrow & G_{1}(O) \\
\left(x_{1}, \ldots, x_{n}\right) & \mapsto & \prod_{i=1, \ldots, n} g_{i} f_{i}\left(x_{i}\right) g_{i}^{-1}
\end{array}
$$

soit surjective. 
Comme la $R$-équivalence est triviale pour les tores $k$-rationnels, on en déduit immédiatement que $G_{1}(O) \subset R G(K)$, c'est-à-dire le lemme 2.4 dans ce cas.

3.2. Techniques de déformation de Kollár. La proposition de Madore présente plusieurs avantages, elle est élémentaire et la seule hypothèse est la projectivité; en particulier, il n'y a pas d'hypothèse de lissité. Avec des techniques de déformation, Kollár a démontré le résultat suivant.

Théorème 3.2 ([K2], théorème 2$)$. On suppose A hensélien. Soit $\mathfrak{X}$ un A-schéma propre et lisse. On suppose que la fibre spéciale $X / k=\mathfrak{X} \times{ }_{A} k$ est séparablement rationnellement connexe, i.e. il existe une variété $V / k$ et un morphisme $F: V \times \mathbb{P}_{k}^{1} \rightarrow X$ tel que le morphisme

$$
F(., 0) \times F(., \infty): V \rightarrow X \times X
$$

est dominant et séparable. Alors la spécialisation $\mathfrak{X}(A) \rightarrow X(k)$ induit une bijection

$$
\mathfrak{X}(F) / R \stackrel{\sim}{\longrightarrow} X(k) / R .
$$

En particulier, une variété séparablement unirationnelle (i.e. il existe un morphisme dominant et séparable d'un ouvert d'un espace affine vers cette variété) est séparablement rationnellement connexe, ce qui est le cas des groupes réductifs et de leurs compatifications lisses ([B], th. 18.2). Vu la proposition 1.8 le théorème 3.2 entraîne la théorème principal en caractéristique nulle. On a aussi une autre conséquence due à la compactification de De Concini et Procesi et au fait qu'elle se relève à $A$ ([CS, Theorem 3.13).

Corollaire 3.3. On suppose $A$ hensélien, $\operatorname{car}(k) \neq 2$ et que $\mathfrak{G}$ est un $A$-schéma en groupes adjoints à fibre spéciale anisotrope. Alors la spécialisation $\mathfrak{G}(A) \rightarrow G(k)$ induit une bijection

$$
\mathfrak{G}(F) / R \stackrel{\sim}{\longrightarrow} G(k) / R .
$$

Le gain obtenu avec le théorème de Kollár est donc l'injectivité du morphisme de spécialisation en caractéristique mixte.

3.3. Commentaire sur l'hypothèse de caractéristique. L'hypothèse de caractéristique distincte de 2 intervient dans la compactification magnifique de De Concini-Procesi décrite dans l'article [CS] dans le cadre assez général comprenant le cas de schémas en groupes adjoints non nécessairement déployés définis sur un anneau de valuation discrète, ce qui nous a permis de définir un morphisme de spécialisation en caractéristique mixte. Pour un $k$-groupe adjoint déployé, on sait que la compactification de De Concini-Procesi existe aussi en caractéristique 2 suivant Strickland $[\mathrm{S}]$. Nous ignorons si les résultats de [CS] (et donc notre théorème de spécialisation) s'étendent lorsque car $(k)=2$.

\section{REMERCIEMENTS}

Ce travail a bénéficié de discussions bienvenues avec Michel Brion, Antoine Chambert-Loir, Jean-Louis Colliot-Thélène et M.S. Raghunathan, c'est avec grand plaisir que je les remercie. 


\section{RÉFÉRENCES}

[BM] E. Bierstone et P.D. Milman, Canonical desingularization in characteristic zero by blowing up the maximum strata of a local invariant, Invent. Math. 128 (1997), 207-302. MR 98e:14010

[Bo] A. Borel, Linear algebraic groups, seconde édition, Graduate Texts in Mathematics 126, Springer-Verlag. MR 92d:20001

[BLR] S. Bosch, W. Lütkebohmert et M. Raynaud, Néron Models, Ergebnisse der Mathematik und ihrer Grenzgebiete 21 (1990) Springer-Verlag. MR 91i:14034

[BrT] F. Bruhat et J. Tits, Groupes réductifs sur un corps local II, Publ. Math. IHES 60 (1984). MR 86c:20042

[CM] V. Chernousov et A.S. Merkurjev, R-equivalence in spinor groups, J. Amer. Math. Soc. 14 (2001), 509-534. MR 2002d:14074

[CTS1] J-L. Colliot-Thélène et J.-J. Sansuc, La R-équivalence sur les tores, Ann. Scient. ENS, vol. 10 (1977), 175-230. MR 56:8576

[CTS2] J-L. Colliot-Thélène et J.-J. Sansuc, Principal homogeneous spaces under flasque tori : applications, J. of Alg. 106 (1987), 148-205. MR 88j:14059

[CS] C. De Concini et T.A. Springer, Compactification of symmetric varieties, Transform. Groups 4 (1999), 273-300. MR 2000f:14079

[EH] S. Encinas et H. Hauser, Strong resolution of singularities in characteristic zero, preprint (2002), ArXiv: math.AG/0211423.

[G] P. Gille, La R-équivalence sur les groupes algébriques réductifs définis sur un corps global, Publ. Math. I.H.E.S. 86 (1997), 199-235. MR 99c:20066

[GD] A. Grothendieck et J. Dieudonné, Éléments de Géométrie Algébrique IV, Pub. Math. IHES. 20 (1964), 24 (1965), 28 (1966), 32 (1967). MR 30:3885. MR 33:7330, MR 36:178

[H] H. Hironaka, Resolution of singularities of an algebraic variety over a field of characteristic zero. I, II., Ann. of Math. 79 (1964), 109-203 et 205-326. MR 33:7333

[K1] J. Kollár, Rationally connected varieties over local fields, Ann. of Math. 150 (1999), 357-367. MR 2000h:14019

[K2] J. Kollár, Specialization of zero-cycles, prépublication (2002), math.AG/0205148.

[M] Yu. I. Manin, Cubic forms: algebra, geometry, arithmetic, seconde édition, North-Holland (1986). MR 87d:11037

[P] G. Prasad, Elementary proof of a theorem of Bruhat-Tits-Rousseau and of a theorem of Tits, Bull. Soc. Math. France 110 (1982), 197-202. MR 83m:20064

[R] M. S. Raghunathan, Principal bundles admitting a rational section, Invent. Math. 116 (1994), 409-423. MR 95f:14093

[S] E. Strickland, A vanishing theorem for group compactifications, Math. Ann. 277 (1987), 165-171. MR 88b:14035

[SGA3] Séminaire de Géométrie algébrique de l'I.H.E.S., 1963-1964, schémas en groupes, dirigé par M. Demazure et A. Grothendieck, Lecture Notes in Math. 151-153, Springer (1970). MR 43:223a

[T] J. Tits, Strongly inner anisotropic forms of simple algebraic groups, J. Algebra 131 (1990), 648-677. MR 91g:20069

[V] V. E. Voskresenskiǐ, O privedennoi gruppe Uaitheda prostoi algebry (Sur le groupe de Whitehead d'une algèbre simple), Uspekhi Mat. Nauk 32 (1977), 247-248. MR 58:16677

UMR 8628 du C.N.R.S., Mathématiques, BÂtiment 425, Université de Paris-Sud, F91405 Orsay, France

E-mail address: gille@math.u-psud.fr 